\title{
Optical Fibre Grating Refractometers for Resin Cure Monitoring
}

\author{
S J Buggy, E Chehura, S W James and R P Tatam \\ Engineering Photonics Group \\ School of Engineering \\ Cranfield University \\ Cranfield \\ Bedfordshire \\ MK43 0AL \\ r.p.tatam@cranfield.ac.uk
}

\begin{abstract}
The use of fibre grating refractometers as a means of monitoring the cure of a UVcured epoxy resin is presented. The wavelength shift of the attenuation bands of a long period grating and the spectral response of a tilted fibre Bragg grating sensor were measured simultaneously during the cure of the resin and compared with measurements made using a fibre optic Fresnel based refractometer. The results showed a good correlation $\left(6 \times 10^{-3}\right.$ rius $)$ and illustrate the potential of the techniques for non-invasive composite material cure monitoring.
\end{abstract}

\section{Introduction}

Many fibre optic techniques have been reported for the in-situ monitoring of the cure of epoxy resins [1]. They offer clear advantages over traditional monitoring techniques, arising from their inherently small dimensions and high sensitivity. Spectroscopic methods predominantly use the fibre as a means for delivering the light to and from a sample [1]. However, direct interaction of the propagating mode with the surrounding environment has also been reported, achieved by etching or side polishing the optical fibre to remove the cladding and thus expose the evanescent tail of the core mode field [2]. For applications requiring embedding of the optical fibre, such as the monitoring of the cure of composite materials, techniques that do not require removal of the fibre cladding are attractive. In-fibre grating structures are capable of coupling light from the core of the fibre into the cladding, where the interaction of the cladding modes with the external environment enables the measurement of the surrounding refractive index without the need for etching or side polishing of the fibre. Two in-fibre grating structures which act as core to cladding mode couplers are considered here, the long period grating (LPG) and the tilted fibre Bragg grating (TFBG). An LPG produces a discrete set of attenuation bands in the transmission spectrum of the optical fibre [3]. The refractive index sensitivity of LPGs has been exploited for a range of applications, including, chemical concentration sensing [4], liquid level sensing [5] and as a means of forming a tuneable spectral filter [6]. A TFBG produces numerous resonances in the transmission spectrum of the optical fibre, the monitoring of which has been used to demonstrate a refractometer [7], again without the requirement for etching, side polishing [8] or tapering [9] the fibre. 


\subsection{Long Period Gratings}

A long period grating (LPG) consists of a periodic modulation of the refractive index of the core of an optical fibre. The period of the modulation is typically in the range $10 \mu \mathrm{m}$ to $1000 \mu \mathrm{m}$, and promotes coupling between co-propagating modes of the optical fibre. In the case of single mode fibre, the coupling takes place between the guided mode and co-propagating cladding modes. Efficient coupling is possible to only a subset of these cladding modes [3]. As the cladding modes are poorly guided and suffer from high attenuation, the transmission spectrum of an optical fibre containing an LPG contains a number of attenuation bands, each corresponding to coupling to a different cladding mode. The phase matching wavelengths are governed by the expression [10],

$$
\lambda_{i}=\left|n_{\text {eff_co }}(\lambda)-n_{\text {eff_cl }}^{i}(\lambda)\right| \Lambda
$$

where $\lambda_{\mathrm{i}}, \mathrm{n}_{\text {eff_co }}, \mathrm{n}_{\text {eff_cl }}^{\mathrm{i}}$ and $\Lambda$ are the $\mathrm{i}^{\text {th }}$ cladding mode resonance wavelength, effective index of the core, effective index of the $i^{\text {th }}$ cladding mode, and the grating period respectively. The refractive index sensitivity of LPGs arises from the dependence of the coupling wavelength upon the effective index of the cladding mode. The response of an LPG to refractive index is manifested as a shift in the central wavelength of the attenuation bands; this is shown in figure 1. The higher order modes show an increased sensitivity, and this is enhanced as the refractive index approaches that of the cladding. When the refractive index matches that of the cladding, the cladding modes are no longer supported as the cladding appears to be of infinite thickness. Further increase of the refractive index leads to the bands reappearing, corresponding to the existence of leaky modes, but at a longer wavelength and with significantly reduced refractive index sensitivity [10].

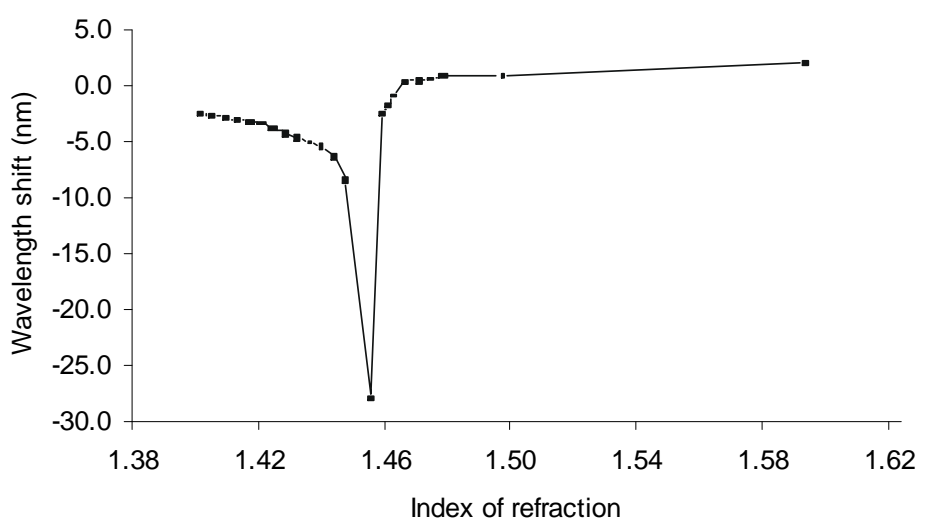

Figure 1. Plot of the wavelength shift of an attenuation band against the index of refraction of the material surrounding an LPG of period $400 \mu \mathrm{m}$, written in boron-germanium co-doped fibre with a cutoff wavelength of $650 \mathrm{~nm}$ [5].

The LPG response to changes in the surrounding refractive index, shown in figure 1, was characterised by immersing the section of fibre containing the LPG in a series of Cargille refractive index oils of differing refractive index. The refractive index at the wavelength of operation was calculated using the Sellmeier equation. The wavelength response was then fitted with a $6^{\text {th }}$ order polynomial to provide a function relating wavelength shift to refractive index. 


\subsection{Tilted Fibre Bragg Gratings}

A standard fibre Bragg grating (FBG) consists of a refractive index modulation in the core of an optical fibre that acts to couple the fundamental forward propagating mode to the contra-propagating core mode. A tilted FBG (TFBG) [11,12] consists of a refractive index modulation that is purposely tilted or blazed relative to the fibre axis in order to enhance coupling between the forward-propagating core mode and contra-propagating cladding modes, as illustrated in figure 2 . The contra-propagating cladding modes are poorly guided and attenuate rapidly and are therefore not observable in reflection but are observed as numerous resonant bands in the transmission spectrum of the TFBG. A typical transmission spectrum is shown in figure 3 .

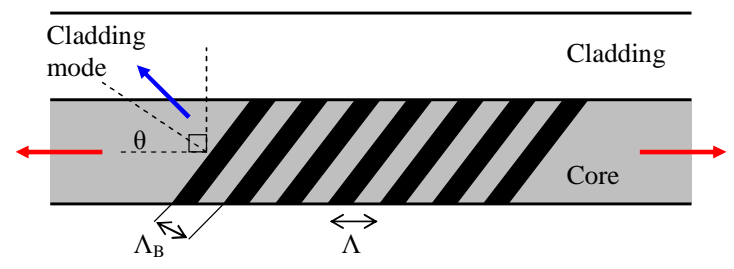

Figure 2. Schematic diagram of a tilted fibre Bragg grating (TFBG). $\Lambda$; grating period, $\Lambda_{\mathrm{B}}$; blaze period and $\theta$; blaze angle.

The spectral response of the TFBG is governed by the phase matching condition:

$$
\lambda_{i}=\left(n_{\text {eff_co }}+n_{\text {eff_cl }}^{i}\right) \cdot \Lambda_{\mathrm{B}} \text {, where } \Lambda_{\mathrm{B}}=\frac{\Lambda}{\cos \theta}
$$

where $\lambda_{\mathrm{i}}, \mathrm{n}_{\text {eff_co }}, \mathrm{n}_{\text {eff_cl }}^{\mathrm{i}}, \theta, \Lambda$ and $\Lambda_{\mathrm{B}}$ are the $\mathrm{i}^{\text {th }}$ cladding mode resonance wavelength, effective index of the core, effective index of the $i^{\text {th }}$ cladding mode, tilt angle, grating period and blaze period respectively.

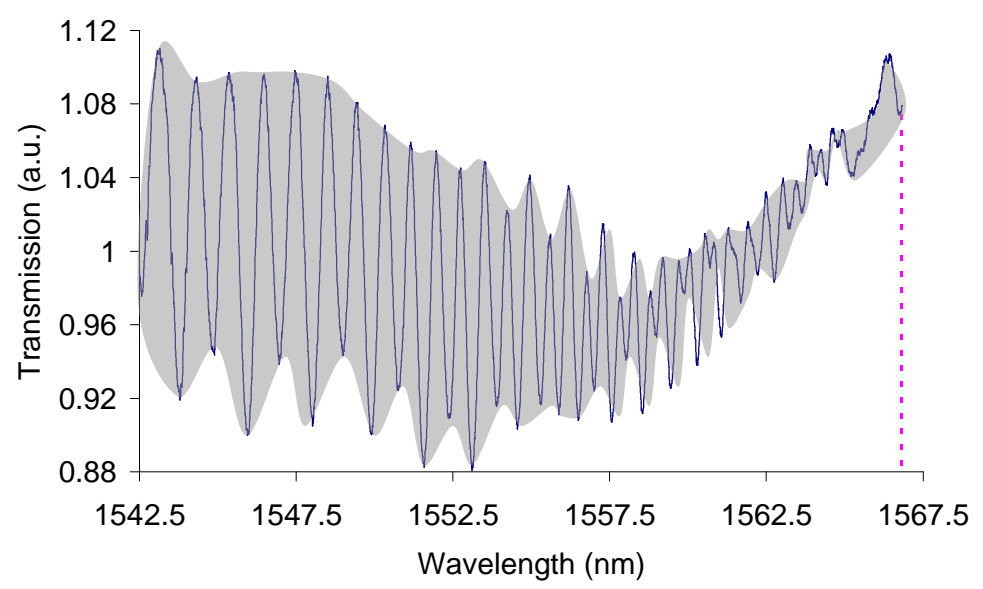

Figure 3. Transmission spectrum of a TFBG of length $5 \mathrm{~mm}$ and blaze angle $4.5^{\circ}$, fabricated in borongermanium co-doped optical fibre (Fibercore PS1250). The dotted line indicates the position of the Bragg wavelength. The shaded area illustrates the envelope technique used for area calculation.

The TFBG spectral resonances are more complex and require a different method of analysis compared to that of the LPG. One method by which this may be achieved is by constructing an envelope that completely surrounds the spectral resonances [7]. The area that is enclosed by the envelope varies with changes in the refractive index of the surrounding environment. By monitoring this change and relating it to the refractive index it is possible to use the TFBG as a refractometer. The 
TFBG response to changes in surrounding refractive index, shown in figure 4 , was characterised by immersing the section of fibre containing the TFBG in a series of Cargille refractive index oils of differing refractive index. The refractive index at the wavelength of operation was calculated using the Sellmeier equation The wavelength response was then fitted with a $3^{\text {rd }}$ order polynomial to generate a function relating wavelength shift to refractive index.

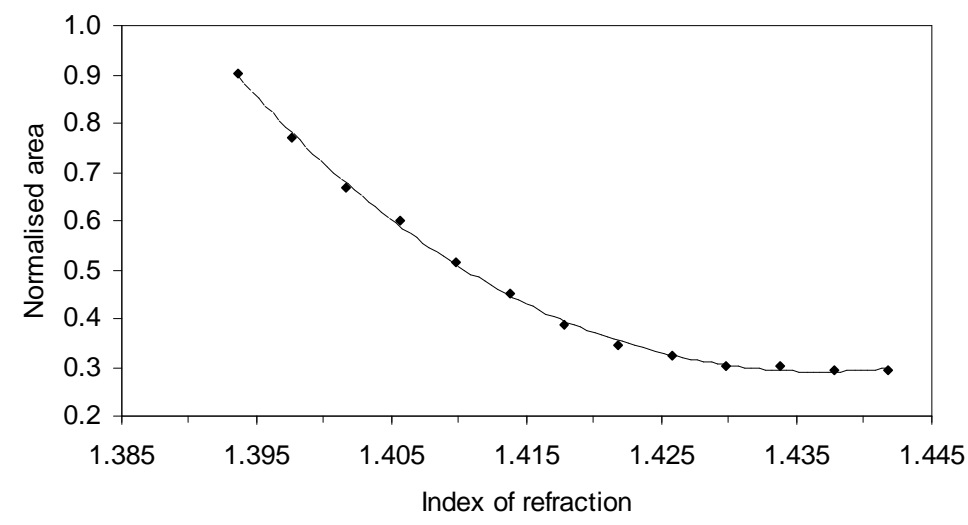

Figure 4. Normalised area of the envelope of the TFBG resonances (with reference to that when the TFBG is in air) plotted as a function of the refractive index of the surrounding medium.

In this paper, the use of LPGs and TFBGs as resin cure monitoring sensors is investigated. The index of refraction of a polymerising resin changes as the molecular density increases and as the atomic bonds involved in the polymerisation processes are altered [13]. The sensitivities of TFBGs and LPGs to changes in refractive index are exploited with the aim of developing a method of measuring the degree of cure. To aid cross comparison of results, a Fresnel reflection based refractometer has been designed and implemented [14].

\section{Experiment.}

A TFBG of length $5 \mathrm{~mm}$ and blaze angle $4.5^{\circ}$ was fabricated in boron-germanium co-doped optical fibre, Fibercore PS1250 with a cut-off wavelength of $1240 \mathrm{~nm}$, using the near-field interference pattern of a tilted phase-mask. The photosensitivity of the fibre was enhanced by pressurizing it in hydrogen for a period of 2 weeks at a pressure of 150 bar at room temperature. The phase mask was illuminated with a UV beam at a wavelength of $248 \mathrm{~nm}$ and with an average power of $40 \mathrm{~mW}$. The TFBG was interrogated by coupling the output from a super-luminescent diode of bandwidth $60 \mathrm{~nm}$ into the optical fibre, and monitoring the transmission using a scanning fibre Fabry-Perot interferometer of free spectral range $43 \mathrm{~nm}$ and of finesse 900 [15].

To investigate the LPG response to the refractive index change of the resin at different wavelengths, LPGs of length $40 \mathrm{~mm}$ and $400 \mu \mathrm{m}$ period were fabricated in two different fibre types, one with a cut off wavelength of 650nm (Fibercore SM750) and the other with a cut off of $1275 \mathrm{~nm}$ (Optical Fibres 1310). The fibre was photosensitised by pressurising it in hydrogen for a period of 2 weeks at a pressure of 150 bar at room temperature. The fibre was then placed behind an amplitude mask that was illuminated by a UV laser beam at a wavelength of $266 \mathrm{~nm}$, provided by an injectionseeded frequency-quadrupled Nd:YAG laser.

The transmission spectrum of the fibre with the shorter cut-off wavelength, shown in figure 5, was monitored by coupling the output from a tungsten-halogen white light source into the fibre and 
coupling the transmitted light to a CCD spectrometer (Ocean Optics S2000) with a resolution of $0.3 \mathrm{~nm}$. The transmission spectrum of the fibre with the longer cut-off wavelength was monitored using an Advantest Q8381 spectrum analyser with a resolution of $0.1 \mathrm{~nm}$.

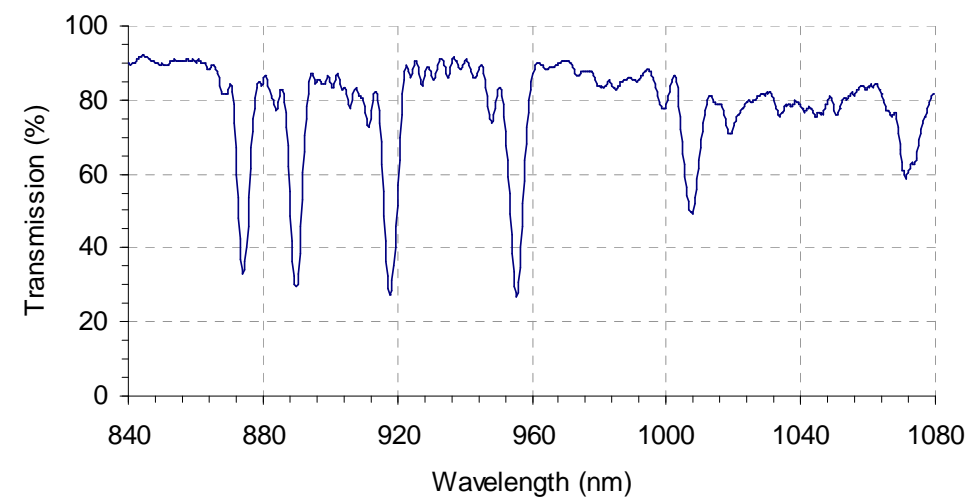

Figure 5. Transmission spectrum of an LPG of length $40 \mathrm{~mm}$ and of period of $400 \mu \mathrm{m}$, fabricated in an optical fibre with a cut-off wavelength of 650nm (Fibercore SM750).

The responses of the fibre grating based refractometers to the refractive index change were compared with refractive index measurements made using a Fresnel based refractometer [14]. Two Fresnel refractometers were constructed, operating at wavelengths of $833 \mathrm{~nm}$ and $1575 \mathrm{~nm}$. This was to aid correlation with the wavelength regions used by the fibre gratings and to reduce the differences introduced by the dispersion of the resin. The Fresnel approach is complementary to the grating sensors in offering a highly localised measurement of the refractive index change at the end of a fibre that can be readily calibrated, as described below. However, this approach is not as appropriate for applications where multiplexing of several sensors is required, or where a longer gauge length sensor is necessary.

\subsection{Fresnel Refractometer}

The experimental configuration is shown in figure 6. The output from a laser diode operating at the appropriate wavelength was intensity modulated at a frequency of $270 \mathrm{~Hz}$, and coupled into a network of $3 \mathrm{~dB}$ directional couplers. The Fresnel reflection from the fibre/resin interface was monitored using photodiode pd1. In the second arm, photodiode pd2 monitored the reflection from the air/fibre interface, acting as an intensity reference to aid the normalisation of the signal and to account for any change in power coupled into the optical fibre from the laser diode. The outputs from the photodiodes were monitored using lock-in amplifiers. To minimize any contributions from unwanted Fresnel reflections from the unused port of the $3 \mathrm{~dB}$ coupler, micro bends were induced in the fibre to form a high attenuation return path for the reflections.

The refractive index determined using the refractometer is given by [14]

$$
\begin{gathered}
n=n_{\text {eff_co }} \times \frac{1-\frac{\Delta}{\sqrt{R}}}{1+\frac{\Delta}{\sqrt{R}}} \\
\Delta=\frac{n_{\text {eff_c }} c o^{-n_{a}}}{n_{\text {eff_c }} c o^{+n_{a}}}
\end{gathered}
$$




$$
R=\frac{V_{\text {air }}}{V_{\text {epoxy }}}
$$

where, $n_{\text {eff } c o}$ is the effective refractive index of the fibre mode, $n_{a}$ is the refractive index of air given as 1.0002739 [14], $V_{\text {air }}$ is the voltage produced by photodiode $\mathrm{pd}_{2}$, obtained for the reflection from the fibre/air interface and $V_{\text {epoxy }}$ is the voltage produced by photodiode $\mathrm{pd}_{1}$, corresponding to the reflection from the fibre/resin interface.

A UV cured epoxy resin, EpoTek OG134, with an uncured refractive index of 1.4163, measured at $589 \mathrm{~nm}$ [17], was used as the test resin. A UV lamp, with output power 4W and with a spectral peak at $365 \mathrm{~nm}$, was positioned to provide a fluence of $6.57 \mathrm{~mW} / \mathrm{cm}^{2}$ in order to cure the resin slowly and to allow the reaction to be recorded using the relatively low sampling rate achievable using the OSA. The LPG and TFBG spectra and the reflection signal from the Fresnel probe were recorded at $0.5 \mathrm{~s}$ intervals during the cure.

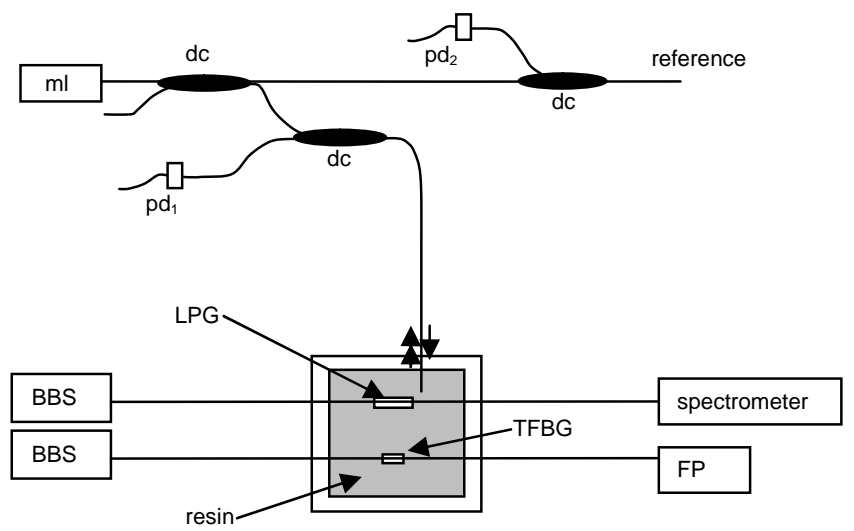

Figure 6. Experimental configuration. ml, modulated laser diode light source; pd, photodiode; dc, directional coupler; BBS, broadband source; FP, scanning Fabry-Perot interferometer; LPG, long period grating; TFBG, tilted fibre Bragg Grating.

\section{Results}

Figure 7 shows the refractive index of the curing resin measured by both of the Fresnel based refractometers (solid line $-833 \mathrm{~nm}$ and dashed line $-1575 \mathrm{~nm}$ ). The datum is the time at which the UV lamp is switched on. At this time a clear decrease in refractive index was observed. This is due to an exothermic reaction initiated by the incident UV light. The temperature of the resin was measured with a thermocouple, placed in the resin, which indicated a $6.5^{\circ} \mathrm{C}$ increase in temperature over the first 5 minutes. It was also noted that there was an ambient temperature increase. This was measured with a thermocouple placed close to the sample. The thermocouple measurements are shown in figure 8 .

The measurements indicating the evolution of the refractive index made at the two wavelengths show good agreement. After the initial decrease in the refractive index, a rapid increase is measured, indicating the reaction process. The rate of change of refractive index reduces after 90 minutes. The difference between the measurements made at the two wavelengths may be due to the dispersion of the resin. 


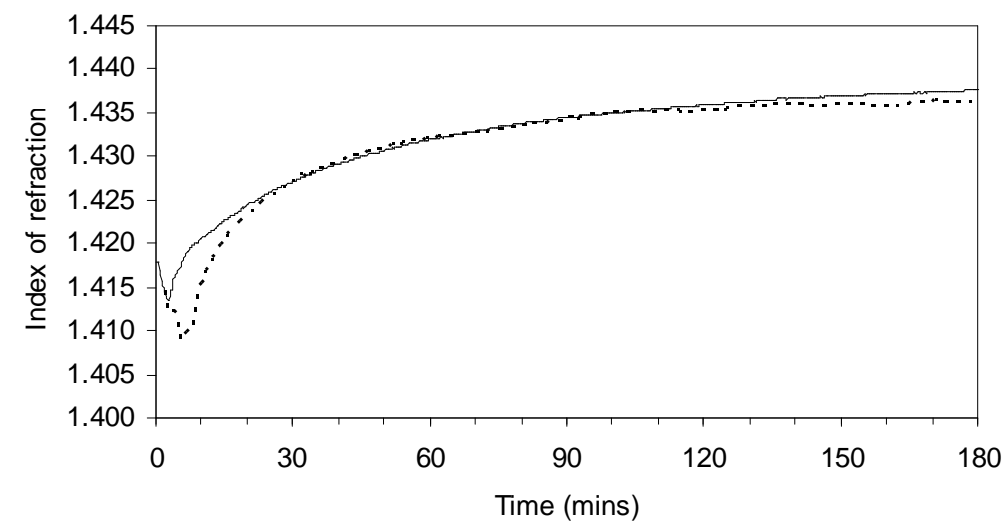

Figure 7. Refractive index change measured during the cure of the OG-134 epoxy resin, determined from the measurements made using the Fresnel refractometers operating at $833 \mathrm{~nm}$ (solid) and $1575 \mathrm{~nm}$ (dashed)

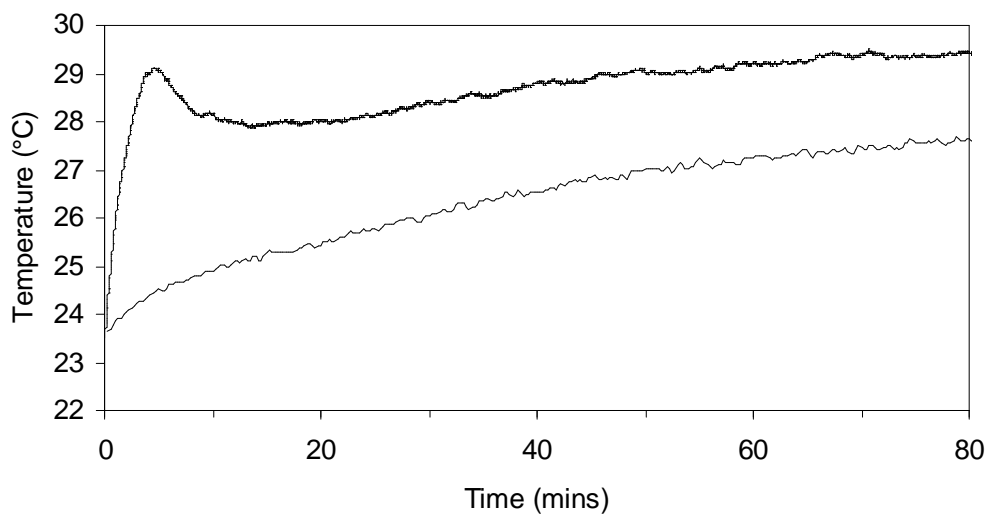

Figure 8. Temperature of the OG-134 epoxy resin measured during cure by two K-type thermocouples; one placed in the sample (solid) and the second placed close to the sample (dashed). An exothermic peak is evident shortly after the UV lamp was turned on.

To aid comparison of the LPG's response to the resin during cure, a simple model was used. The model uses the Gloge weakly guiding approximation [18] to calculate the effective refractive index of the core mode. The standard dispersion equation for a step index fibre [19] was used to calculate the effective indices of the cladding modes. The central wavelengths of the attenuation bands may then be determined using equation (1). The refractive index measured during the cure of the resin using the Fresnel system was then input to the model to calculate the corresponding change in the cladding mode effective index, and thus allow prediction of the change in the LPG attenuation band wavelength. Figure 9 shows the prediction of the central wavelength of the longest wavelength $\left(5^{\text {th }}\right)$ attenuation band of an LPG fabricated in an optical fibre with a cut-off wavelength of $650 \mathrm{~nm}$, of length $40 \mathrm{~mm}$ and with a $400 \mu \mathrm{m}$ period. Shown here also is the actual response of the longest wavelength band of an LPG fabricated in the lower cut-off fibre (Fibercore SM750) of length 40mm and with a period of $400 \mu \mathrm{m}$. The close agreement with the model shows the potential for using an LPG as a cure monitoring sensor. The contributions of strain and temperature to the measured wavelength shift are not included in the simplified model. Transverse strain is known to develop during the cure for some resin systems [20], but has not yet been determined for the UV resin used here. 


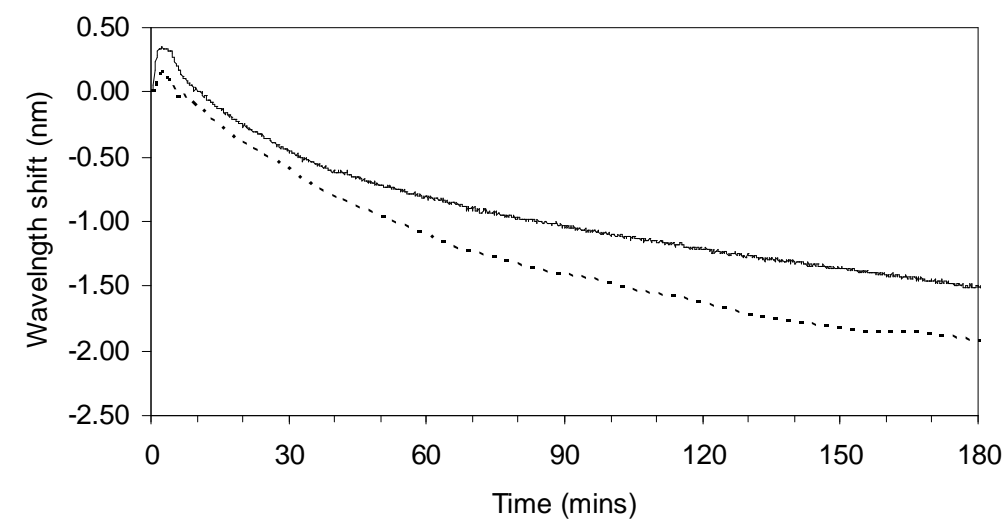

Figure 9. The experimental (solid) and theoretical (dashed) wavelength response of the $5^{\text {th }}$ attenuation band of an LPG (central wavelength at $940 \mathrm{~nm}$ ), of length $40 \mathrm{~mm}$ and of period $400 \mu \mathrm{m}$ fabricated in a low cut-off wavelength fibre (SM750), during the cure of the OG-134 epoxy resin.

Using the calibration method discussed in section 1.1, the wavelength responses of the LPGs are related to refractive index. Figure 10 shows the refractive index calculated using the fifth attenuation bands of both LPGs (SM750 and OF1310 fibre). At both wavelengths the initial decrease in refractive index, observed when the UV lamp is switched on, is followed by a rapid positive change in refractive index. As exhibited by the Fresnel refractometer, the LPGs show a decrease in the rate of change of refractive index after 90 minutes. The discrepancies may again be attributed to dispersion effects and to errors induced in the calibration, where the thermo-optic change in refractive index of the resin was assumed to be negligible. The results obtained using both the Fresnel technique and the LPG based method indicates that the onset of the positive refractive index change is delayed when the measurement is made at the longer wavelength. This may point to the dispersive properties of the resin. The refractive index of the resin at the shorter wavelengths $(\sim 800 \mathrm{~nm})$ approaches the high sensitivity regions of the refractometers before that of the longer wavelengths $(\sim 1550 \mathrm{~nm})$.

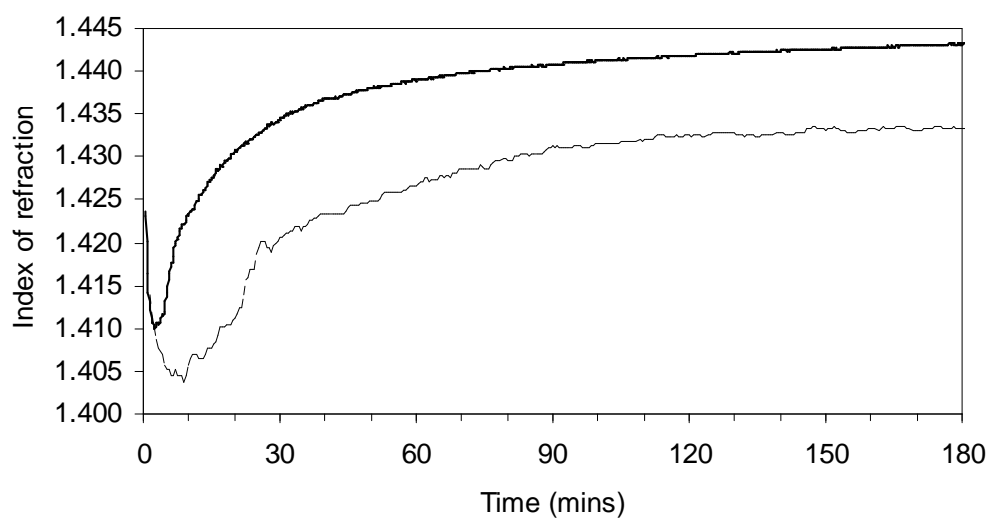

Figure 10. The evolution of the refractive index during cure of the OG-134 epoxy resin, determined from the wavelength shift of the $5^{\text {th }}$ attenuation bands of the LPG based refractometers at $940 \mathrm{~nm}$ (solid) and 1545nm (dashed).

Tables 1 and 2 compare the measurements from the LPG and Fresnel techniques in the two wavelength .regimes. 
Table 1. Comparison of the refractive index change exhibited by EpoTek OG134 during cure, determined from the Fresnel (833nm) and LPG (fabricated in the PS750 fibre) methods. ( $\Delta$ is the difference between the measurements made using the two methods).

\begin{tabular}{|l|lll|}
\hline $\begin{array}{r}\text { Optical } \\
\text { technique }\end{array}$ & Fresnel & LPG & $\Delta$ \\
\hline Cure state & & & \\
\hline Wet (initial) & 1.4178 & 1.4237 & 0.0059 \\
Cured (final) & 1.4376 & 1.4432 & 0.0056 \\
\hline
\end{tabular}

Table 2. Comparison of the refractive index change exhibited by EpoTek OG134 during cure, determined from the Fresnel $(1575 \mathrm{~nm})$ and LPG (fabricated in the OF1310 fibre) methods. ( $\Delta$ is the difference between the measurements made using the two methods).

\begin{tabular}{|l|lll|}
\hline $\begin{array}{r}\text { Optical } \\
\text { technique }\end{array}$ & Fresnel & LPG & $\Delta$ \\
\hline Cure state & & & \\
\hline Wet (initial) & 1.4184 & 1.4190 & 0.0006 \\
Cured (final) & 1.4365 & 1.4332 & -0.0033 \\
\hline
\end{tabular}

The refractive index change determined over the cure cycle by the TFBG is shown in figure 11. The form of the curve agrees well with the results from the Fresnel and LPG based refractometers. The refractive index starts to increase 5 minutes after the UV lamp is switch on. The TFBG sensor indicates a refractive index of 1.437 when the resin is fully cured, achieved after 180 minutes. This agrees, to within $5 \times 10^{-4}$ rius (refractive index units), with the measurement from the Fresnel refractometer operating at $1575 \mathrm{~nm}$. The calibration discussed in section 1.2 revealed that the sensitivity of the TFBG decreases rapidly for refractive indices greater than 1.43 , and it is apparent that the response curve starts to flatten after 60 minutes as the TFBG starts to lose sensitivity. All three sensor types show that most of the reaction takes place in the first 90 minutes, after which there is a gradual reduction in the rate of change of refractive index. The reaction is almost complete after 2 hours. 


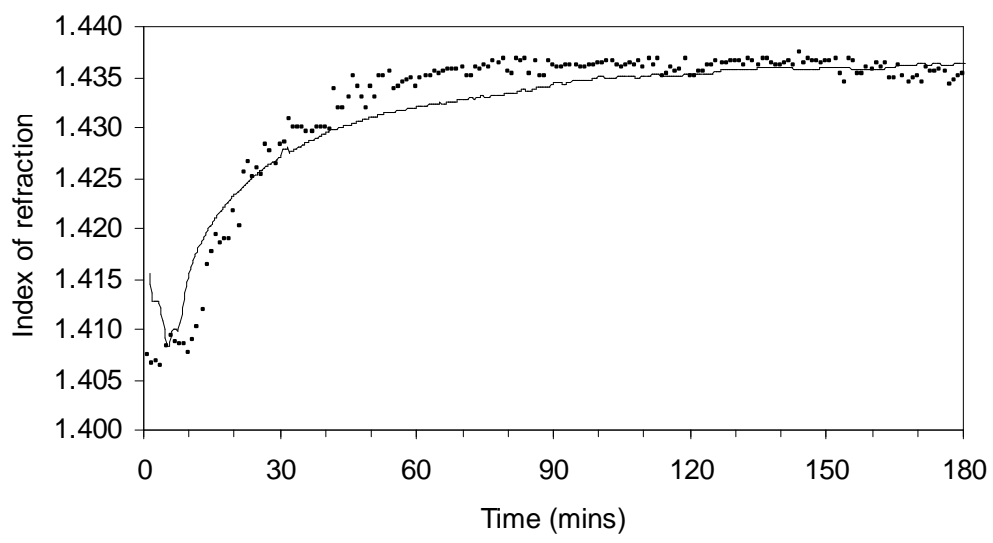

Figure 11. Tilted Fibre Bragg Grating (TFBG) calibrated response to refractive index change at $1550 \mathrm{~nm}$ for the OG-134 UV cured epoxy during cure( $(\mathbf{)})$ The Fresnel response at $1575 \mathrm{~nm}$ (dashed) is also shown for comparison.

The performances of the different techniques are summarised in table 3 . The discrepancy between results obtained using the Fresnel and grating based refractometers may be attributed to the temperature and strain sensitivities of the LPG and TFBG sensors, as mentioned previously. The thermo-optic effect may also have an influence on the resonant wavelength, equations (1) and (2). This effect is negligible for the Fresnel technique. Investigation into the influence of these effects is the subject of current work.

Table 3. Comparison of refractometer methods (depending on fibre type).

\begin{tabular}{lll}
\hline Refractometer & Gauge Length & Sensitivity Range \\
\hline LPG & $10-1000 \mathrm{~mm}$ & $1.400-1.456$ \\
TFBG & $0.1-12 \mathrm{~mm}$ & $1.405-1.437$ \\
Fresnel & $2-10 \mu \mathrm{m}$ & Air-1.456 \\
\hline
\end{tabular}

\section{Summary}

In summary, optical fibre refractometers have been employed to monitor the refractive index changes occurring in a curing epoxy resin. The core-cladding mode coupling resonances of a tilted fibre Bragg grating (TFBG) and of an LPG have been shown to be sensitive to the cure of an epoxy resin. The measurements made using the grating refractometers were compared with those made using a fibre optic Fresnel reflection based technique, and were found to be in agreement to within $6 \times 10^{-4}$ rius. The grating based refractometers are sensitive to temperature and strain, which must be decoupled to accurately measure refractive index. These techniques may be used with cure-kinetic models to characterise and monitor the cure of resins and fibre reinforced resin systems [20].

The authors gratefully acknowledge the support for this work from the Engineering and Physical Sciences Research Council (EPSRC), UK.

\section{References}

[1] Degamber B and Fernando G F 2002 Process monitoring of fiber-reinforced polymer composites MRS Bulletin 27 370-80 
[2] Takeo T and Hattori H 1982 Optical Fiber Sensor for Measuring Refractive Index Japan J. Appl. Phys 21 1509-12

[3] James S W and Tatam R P 2003 Optical Fibre Long Period Grating Sensors: Characteristics and Application_Measurement Science and Technology 14 49-61

[4] Allsop T Zhang L and Bennion I 2001 Detection of organic aromatic compounds in paraffin by a long-period fiber grating optical sensor with optimized sensitivity Optics Communications 191 181-90

[5] Khaliq S James S W and Tatam R P 2001 Fibre optic liquid level sensor using a long period grating Optics Letters 26 1224-1226,

[6] Min-Suk Kwon and Sang-Yung Shin 2004 Tunable notch filter using a thermooptic long-period grating Journal of Lightwave Technology 22 1968-75

[7] Laffont G and Ferdinand P 2001 Tilted short-period fibre-Bragg-grating-induced coupling to cladding modes for accurate refractometry Measurement Science and Technology 12 765-70

[8] Asseh A Sandgren S Ahlfeldt H Sahlgren B Stubbe R and Edwall G 1998 Fiber Optical Bragg Grating Refractometer Fiber and Integrated Optics 17 51-62

[9] Grobnic D Mihailov S J Huimin Ding and Smelser C W 2006 Femtosecond IR laser fabrication of Bragg gratings in photonic crystal fibers and tapers IEEE Photonics Technology Letters 18 160-2

[10] Patrick H J Kersey A D and Bucholtz F 1998 Analysis of the Response of Long Period Fiber Gratings to External Index of Refraction Journal of Lightwave Technology 16 1606-1612

[11] Erdogan T and Sipe J E 1996 Tilted fiber phase gratings Journal of the Optical Society of America A 13 296-313

[12] Brilland L Pureur D Bayon J F and Delevaque E 1999, Slanted gratings UV-written in photosensitive cladding fibre Electronics Letters 35 234-236

[13] Boming Z Dianfu W Shanyi Du and Yinlin S 1999 An investigation of a fiber optic sensor in the composite cure process Smart Materials and Structures 8 515-518

[14] Kim C B and Su C B 2004 Measurement of the refractive index of liquids at 1.3 and 1.5 micron using a fibre optic Fresnel ratio meter Measurement Science and Technology 15 1683-86

[15] Ye C-C Staines S E James S W and Tatam R P 2002 A polarisation maintaining fibre Bragg grating interrogation system for multi-axis strain sensing Measurement Science and Technology 13 1446-9

[16] Weast R C and Selby S M 1967-1968 Handbook of Chemistry and Physics 48th edn E-160

[17] Epoxy Technology Billerica USA www.epotek.com

[18] Gloge D 1971 Weakly guiding fibers Applied Optics 10 2252-2258

[19] Yariv A 1996 Optical electronics in Modern Communications (Oxford University Press)

[20] Chehura E Kazilas M James S W Partridge I K and Tatam R P 2005 Strain development in curing epoxy resin and glass fibre/epoxy composites monitored by fibre Bragg grating sensors in birefringent optical fibre Smart Materials and Structures 14 354-362 